\title{
Clinical Impact of the Early Use of Monoclonal Antibody LY-CoV555 (Bamlanivimab) on Mortality and Hospitalization Among Elderly Nursing Home Patients: A Multicenter Retrospective Study
}

Mohammud M. Alam ${ }^{1}$, Saborny Mahmud ${ }^{2}$, Sandeep Aggarwal ${ }^{3}$, Sawsan Fathma ${ }^{4}$, Naim Al Mahi ${ }^{5}$, Mohammed S. Shibli ${ }^{6}$, Siddiqi M. Haque ${ }^{6}$, Sharothy Mahmud ${ }^{7}$, Ziauddin Ahmed ${ }^{8}$

1. Medicine, Donald and Barbara Zucker School of Medicine at Hofstra/Northwell, Hempstead, USA 2. Medicine, Icahn School of Medicine at Mount Sinai, New York, USA 3. Nephrology, University of Pennsylvania, Philadelphia, USA 4. Internal Medicine, Swedish Hospital, Chicago, USA 5. Genomics Research Center, AbbVie, Chicago, USA 6. Internal Medicine, Glen Cove Center for Nursing and Rehabilitation, Glen Cove, USA 7. Biological Sciences, Barnard College at Columbia University, New York, USA 8. Medicine, Temple University, Philadelphia, USA

Corresponding author: Mohammud M. Alam,dr.tuku@yahoo.com

\section{Abstract}

\section{Importance}

Coronavirus disease 2019 (COVID-19) outbreaks are frequent occurrences in nursing homes and long-term care facilities (LTCFs), resulting in subsequent hospitalization and death.

\section{Rationale}

Virus-neutralizing monoclonal antibodies demonstrate a significant decrease in both viral load and hospital transfer rate among patients with mild-to-moderate COVID-19 infection.

\section{Objective}

To assess the clinical outcomes of COVID-19 patients with mild-to-moderate symptoms in LTCFs who received LY-CoV555 as compared to those who did not receive this treatment.

\section{Design}

Retrospective case-control study and logistic regression analysis.

\section{Setting}

Review began 04/29/2021 Review ended 05/05/2021 Published 05/10/2021

\section{(c) Copyright 2021}

Alam et al. This is an open access article distributed under the terms of the Creative Commons Attribution License CC-BY 4.0., which permits unrestricted use, distribution, and reproduction in any medium, provided the original author and source are credited.

\section{Participants}

Two-hundred forty-six (246) LTCF patients diagnosed with mild-to-moderate COVID-19 infection with positive COVID-19 polymerase chain reaction (PCR) from November 15, 2020, to January 31, 2021.

\section{Methods}

Two-hundred forty-six (246) COVID-19 patients were identified from electronic medical records, out of which 160 cases were exposed to LY-CoV555 treatment (700 mg single dose, intravenous infusion). Eightysix (86) patients were unexposed controls who did not receive monoclonal antibodies, LY-CoV555.

\section{Outcome}

We assessed the odds of death and hospitalization of exposed cases as compared to unexposed controls. Using logistic regression analysis, we also assessed the risk factors associated with these outcomes in the entire sample population.

\section{Results}

The mean age of the entire sample was 82.4 years. Fifty-two percent $(52 \%)$ of patients $(n=129)$ were female and $48 \%(n=117)$ were male. The mean ages of the exposed group and the unexposed group were 81 years and 84 years, respectively. At the end of the study, 92\% (148/160) of the exposed group were alive or not transferred to the hospital as compared to $79 \%(68 / 86)$ patients of the unexposed group (OR 3.23, $95 \% \mathrm{CI}$ : $(1.48,7.31)$, $\mathrm{p}$-value $=0.0032)$. Three percent $(3 \% ; 5 / 160)$ of patients died in the exposed group compared to 
$10 \%(9 / 86)$ of patients who died in the unexposed group $(\mathrm{OR}=0.25,95 \% \mathrm{CI}:(0.1,0.85)$, p-value $=0.0257)$. Four point thirty-seven percent $(4.37 \% ; 7 / 160)$ of patients in the exposed group and $10.46 \%(9 / 86)$ of patients in the unexposed group were transferred to the hospital $(\mathrm{OR}=0.35,95 \% \mathrm{CI}:(0.15,1.08)$, p-value $=$ 0.0793).

\section{Conclusion}

Early treatment with monoclonal antibody LY-CoV555 is associated with decreased mortality among highrisk patients with mild-to-moderate COVID-19 infection in LTCFs. Although not statistically significant, there was a trend towards a lower risk of hospitalization in patients treated with LY-CoV555.

Categories: Infectious Disease, Pulmonology, Public Health

Keywords: covid-19, covid-19 treatment, sars-cov-2, ly-cov555, bamlanivimab, monoclonal antibody, long-term care facility, nursing home, non-hospital setting

\section{Introduction}

The coronavirus disease 2019 (COVID-19) pandemic has spread rapidly, disrupting the lives of millions of people in the world and placing an overwhelming burden on the U.S healthcare system [1]. As of March 31, 2021, there have been over 30 million COVID-19 cases and over 551,000 deaths in the U.S. [2], and nursing home residents and staff account for around 40\% of COVID-19-related deaths [3]. Due to their congregate setting and multiple medical conditions, residents in long-term care facilities (LTCFs) are at high risk for the progression of severe COVID-19 infection, hospitalization, and death. Multiple treatment regimens have been implemented to treat hospitalized patients with COVID-19, including antimalarial drugs [4], antiviral agents [5], anthelmintics [6], immunomodulators [7], glucocorticoids [8], and convalescent plasma [9]. However, treatment options for out-patients with COVID-19 infection are limited. Our group initially used doxycycline and hydroxychloroquine to treat COVID-19 infection in LTCF residents [10] and later used doxycycline alone [11].

The COVID-19 virus enters cells through binding of its spike protein to angiotensin-converting enzyme 2 (ACE2) receptors on target cells [12]. Recently, several studies in animal models with virus-neutralizing monoclonal antibodies for COVID-19 infections have shown promising results [13]. The LY-CoV555 monoclonal antibody binds to the receptor-binding domain of the viral spike protein. Studies demonstrate that utilizing monoclonal antibodies for mild-to-moderate symptoms of COVID-19 infections in outpatient settings reduces the viral load, improves symptoms, and prevents hospitalization [14-16]. Here, we report the clinical findings of high-risk patients in LTCFs with mild-to-moderate COVID-19 who received LYCoV555 treatment.

\section{Materials And Methods}

\section{Study design}

We conducted a retrospective chart review of 246 LTCF residents, diagnosed with mild-to-moderate COVID19 infection with a positive COVID-19 polymerase chain reaction (PCR) test result between November 15, 2020 , and January 31, 2021. Out of the 246, 160 patients were treated with the virus-neutralizing monoclonal antibody LY-CoV555 (Bamlanivimab), a $700 \mathrm{mg}$ single dose infusion over one hour within 48 hours after the initial diagnosis. Patients in the exposed group or patients' families were fully informed about the risks and benefits of LY-CoV555 and provided informed consent before starting this treatment. Safety was assessed in all patients. Eighty-six patients did not receive LY-CoV555 treatment (patients were not prescribed it by their primary care physicians, patients or their families did not consent, or for other reasons). Oversight medical boards and corporate clinical services approved this study.

\section{Statistical analysis}

The data were collected from institutional electronic medical records and saved in a secure portable computer. Data were tabulated as the mean and standard deviation (SD) for continuous variables (e.g., blood urea nitrogen (BUN), C-reactive protein (CRP), D-dimer, etc.) and percentages and numbers for categorical variables (e.g., sex, race, obesity, etc.). Comparisons between patients exposed and not exposed to monoclonal antibodies were conducted using a two-sample t-test for continuous variables and Fisher's exact test for categorical variables. All tests were two-tailed with a statistical significance level of $\alpha=0.05$. Binary logistic regression analysis was conducted to assess the association between risk factors and mortality. We selected our model through stepwise logistic regression and assessed its goodness-of-fit using Akaike Information Criterion (AIC). The R programming language (version 4.0.2; R Foundation for Statistical Computing, Vienna, Austria) was used for all statistical analyses.

\section{Results}

The mean age of all 246 patients was 82.4 years, ranging from 58 to 100 years. Fifty-two percent (52\%) $(n=129)$ of patients were female and $48 \%(n=117)$ were male. The mean ages of the exposed and unexposed 
groups were 81 years and 84 years, respectively. Approximately $5 \%(n=14)$ total patients died and approximately $6 \%(\mathrm{n}=16)$ patients were transferred to the hospital due to clinical deterioration.

Ninety-two percent $(92 \% ; 148 / 160)$ patients of the exposed group were alive or not transferred to hospital as compared to $79 \%(68 / 86)$ of the unexposed patients (OR 3.23, 95\% CI: $(1.48,7.31)$, p-value $=0.0032$ ). Three percent $(3 \% ; 5 / 160)$ patients died in the exposed group as compared to $10 \%(9 / 86)$ patients in the unexposed group $(\mathrm{OR}=0.25,95 \% \mathrm{CI}:(0.1,0.85)$, $\mathrm{p}$-value $=0.0257)$, indicating that patients exposed to LY-CoV555 are statistically significantly less likely to die than the patients who were not exposed to the monoclonal antibody. Exposed patients seem to have lower odds of hospitalization although this finding is not statistically significant at the $5 \%$ level.

Furthermore, the odds of having altered mental status (AMS) is significantly lower in the exposed patient group, whereas higher odds of coronary artery disease (CAD) were observed among patients in the exposed group. Variables such as obesity, congestive heart failure (CHF), chronic obstructive pulmonary disease (COPD), diabetes, and side effects were not associated with exposure status (Table 1).

\begin{tabular}{|c|c|c|c|c|c|}
\hline \multicolumn{2}{|l|}{ Categorical Variables } & $\begin{array}{l}\text { Exposed Patients } \\
(n=160)\end{array}$ & $\begin{array}{l}\text { Unexposed Patients } \\
(n=86)\end{array}$ & $\begin{array}{l}\text { Odds Ratio ( } 95 \% \text { Confidence } \\
\text { Interval) }\end{array}$ & P-value \\
\hline Death & No & $96.9 \%(n=155)$ & $89.5 \%(n=77)$ & $0.25(0.1,0.85)$ & 0.0257 \\
\hline Hospital transfer & No & $95.6 \%(n=153)$ & $89.5 \%(n=77)$ & $0.35(0.15,1.08)$ & 0.0793 \\
\hline \multirow{2}{*}{ Sex } & Female & $48.8 \%(n=78)$ & $59.3 \%(n=51)$ & \multirow{2}{*}{$1.47(0.9,2.58)$} & \multirow{2}{*}{0.1168} \\
\hline & Male & $51.2 \%(n=82)$ & $40.7 \%(n=35)$ & & \\
\hline \multirow{3}{*}{ Race } & Black & $16.9 \%(n=27)$ & $18.6 \%(n=16)$ & \multirow{3}{*}{$0.93(0.37,3.18)$} & \multirow{3}{*}{0.8773} \\
\hline & Hispanic & $8.1 \%(n=13)$ & $8.1 \%(n=7)$ & & \\
\hline & White & $75 \%(n=120)$ & $73.3 \%(n=63)$ & & \\
\hline Altered mental status (AMS) & No & $68.1 \%(n=109)$ & $25.6 \%(n=22)$ & $0.16(0.09,0.29)$ & $<0.0001$ \\
\hline Obesity & No & $55.6 \%(n=89)$ & $48.8 \%(n=42)$ & $0.73(0.45,1.27)$ & 0.2894 \\
\hline Ventilator & No & $95.6 \%(n=153)$ & $94.2 \%(n=81)$ & $0.61(0.23,2.25)$ & 0.6210 \\
\hline Chest pain & No & $76.9 \%(n=123)$ & $77.9 \%(n=67)$ & $1(0.56,1.96)$ & 0.8624 \\
\hline Coronary artery disease (CAD) & No & $41.9 \%(n=67)$ & $57 \%(n=49)$ & $1.76(1.08,3.1)$ & 0.0248 \\
\hline Congestive heart failure (CHF) & No & $72.5 \%(n=116)$ & $62.8 \%(n=54)$ & $0.62(0.37,1.12)$ & 0.1213 \\
\hline $\begin{array}{l}\text { Chronic obstructive pulmonary disease } \\
\text { (COPD) }\end{array}$ & No & $60 \%(n=96)$ & $66.3 \%(n=57)$ & $1.25(0.76,2.25)$ & 0.3375 \\
\hline Cough & No & $11.9 \%(n=19)$ & $18.6 \%(n=16)$ & $1.59(0.83,3.47)$ & 0.1600 \\
\hline Malaise/Weakness & No & $22.5 \%(n=36)$ & $22.1 \%(n=19)$ & $0.95(0.54,1.87)$ & 0.9864 \\
\hline Diarrhea & No & $79.4 \%$ (n=127) & $81.4 \%(n=70)$ & $1.06(0.58,2.17)$ & 0.7157 \\
\hline Diabetes & No & $56.2 \%(n=90)$ & $59.3 \%(n=51)$ & $1.09(0.67,1.92)$ & 0.6487 \\
\hline Side effect & No & $95.6 \%(n=153$ & $90.7 \%(n=78)$ & $0.44(0.18,1.46)$ & 0.236 \\
\hline
\end{tabular}

TABLE 1: Categorical characteristics, clinical outcomes, and clinical features of patients who were exposed and patients who were not exposed to LY-CoV555 treatment, along with their respective odds ratios and $p$-values

Comparing patients who were exposed to LY-CoV555 to patients who were not exposed, after treatment, statistically significant mean differences were observed in the resolution of fever, resolution of shortness of breath (SOB), high lactate dehydrogenase (LDH), age, and pulse oximetry (POX). High LDH along with time to resolution of both fever and SOB tended to be lower on average in the exposed group, whereas the mean POX after treatment is higher in the exposed group. Other variables, such as high D-Dimer, high creatinine, and high troponin, were not significantly different between exposed and unexposed groups (Table 2). 


\section{Cureus}

\begin{tabular}{|c|c|c|c|}
\hline Continuous Variables & Mean in the Exposed Group & Mean in the Unexposed Group & P-value \\
\hline Time to resolution of fever (Days) & $1.98(0.07)$ & $3.9(0.13)$ & $<0.0001$ \\
\hline Time to resolution of shortness of breath (Days) & $2.58(0.07)$ & $3.91(0.13)$ & $<0.0001$ \\
\hline High lactate dehydrogenase (LDH) & $578.33(17.02)$ & $693.09(29.97)$ & 0.0011 \\
\hline Pulse oximetry (POX) after Rx & $95.51(0.27)$ & $92.98(0.76)$ & 0.0023 \\
\hline Age & $81.42(0.68)$ & $84.42(0.89)$ & 0.0084 \\
\hline High D-dimer & $3.41(0.3)$ & $4.1(0.43)$ & 0.1933 \\
\hline High creatinine & $1.65(0.04)$ & $1.75(0.06)$ & 0.2068 \\
\hline High troponin & $0.62(0.11)$ & $0.73(0.19)$ & 0.5861 \\
\hline High ferritin & $516.26(19.57)$ & $503.5(25.11)$ & 0.6891 \\
\hline High regular C-reactive protein (CRP) & $9.22(0.61)$ & $9.05(0.85)$ & 0.8693 \\
\hline Pulse oximetry (POX) before $\mathrm{Rx}$ & $95.12(0.17)$ & $95.09(0.26)$ & 0.9338 \\
\hline High blood urea nitrogen (BUN) & $35.68(1.01)$ & $4 \mathbf{H}^{(1.4}$ & 0.97 \\
\hline
\end{tabular}

TABLE 2: Clinical features with continuous measurements for patients who were exposed to and patients who were not exposed to LY-CoV555 treatment, along with their respective mean values and $p$-values

We used binary logistic regression analysis to assess the association between risk factors and mortality. High D-dimer $(\mathrm{OR}=1.201,95 \% \mathrm{CI}:(1.094,1.319)$, $\mathrm{p}$-value $=0.0001)$, delay in resolution of SOB $(\mathrm{OR}=1.914,95 \%$ CI: $(1.239,2.956)$, p-value $=0.0034)$, chest pain $(\mathrm{OR}=3.709,95 \% \mathrm{CI}:(1.246,11.041)$, $\mathrm{p}$-value $=0.0185)$, diabetes $(\mathrm{OR}=3.587,95 \% \mathrm{CI}:(1.094,11.748)$, $\mathrm{p}$-value $=0.0348)$, and high troponin $(\mathrm{OR}=1.224,95 \% \mathrm{CI}$ : $(1.007,1.488)$, $\mathrm{p}$-value $=0.0424)$ were associated with increased mortality. A higher POX after treatment (OR $=0.783,95 \% \mathrm{CI}:(0.716,0.855)$, $\mathrm{p}$-value $=<0.0001)$ tends to reduce mortality. Other variables, such as BUN, creatinine, CRP, ferritin, and LDH were not associated with mortality.

Four point three-seven $(4.37 \%$; $7 / 160)$ patients who received monoclonal antibodies had infusion-related side effects. These side effects were mild and included generalized weakness, nausea, chills, and diarrhea, and one patient had a mild rash. Other adverse events were self-limiting, and no interventions or hospitalizations were required. Neither anaphylaxis nor serum sickness was noted in the exposed group.

\section{Discussion}

COVID-19 remains a public health emergency. Despite ongoing efforts pertaining to reducing community spread by infection control barriers, social distancing measures, and widespread international vaccination efforts, the treatment of COVID-19 patients with acute illness remains a challenge. While direct cytopathic effects of the COVID-19 virus continue to be matters of investigation, there seems to be a consensus regarding the activation of endogenous immunological [17], inflammatory [18-19], and pro-coagulation pathways [20-21], which lead to adult respiratory distress syndrome (ARDS) and multiorgan failure as a large component of morbidity and mortality in severe COVID-19 syndrome. Thus, managing severe COVID-19 disease becomes complicated as treatment mandates the use of immunosuppressive agents; virus neutralization is not very effective at this stage.

A more reasonable approach is to prevent severe COVID-19 disease in outpatient management, either by active immunization through vaccination [22-23] or by viral neutralization in mild-to-moderate COVID-19 disease, especially in high-risk individuals. Wider availability of early treatment interventions and improving outpatient management of COVID-19 syndrome is critical in order to alleviate the overburdened healthcare system.

Passive transfer of virus-neutralizing antibody, using convalescent plasma, has been attempted in hospitalized patients who are at high risk of progression to severe COVID-19 syndrome [24-25]. A retrospective study showed improved survival in patients with high COVID-19 antibody levels compared with patients with low levels [26]. Although reassuring, a big logistical problem remains to obtain sufficient amounts of antibody titer plasma donors. 
Monoclonal antibodies target the receptor-binding domain on the COVID-19 virus' spike protein that binds to the ACE2 receptor [27], a receptor found on numerous cell types. Viral infection is mediated by the interaction between the viral spike and the ACE2 receptors found on numerous cell types and neutralizing monoclonal antibodies block this event [28]. Two independent groups of investigators have reported the findings of virus-neutralizing monoclonal antibodies. Studies assessing Bamlanivimab (LY-CoV555) and Casirivimab and Imdevimab together (REGN-COV2) - published in 2020 in October and December, respectively - showed a lowering of viral load and reduced rate of hospitalization when given to the patients with confirmed COVID-19 tests [15-16]. These results have led to a US-Food and Drug Administration (FDA) emergency utilization approval of virus-neutralizing antibodies for the treatment of mild-to-moderate COVID-19 infection in adults and pediatric outpatients $>12$ years of age. These therapies are additional tools that can be used to fight COVID-19 infection to prevent hospitalization, severe COVID-19 syndrome, and death.

Our study focuses on a population of elderly LTCF residents who, due to their co-morbidities and proximity to other patients, remain at a higher risk of poor COVID-19 outcomes. We conducted a retrospective analysis from the electronic medical records data of 246 patients in multiple LTCFs in New York. We compared baseline factors and outcome differences in the patients who received the COVID-19 neutralizing antibody, LY-CoV555, to those who did not receive it. LY-CoV555 treatment for patients at high risk for disease progression in LTCFs is associated with lower odds of mortality as compared to patients who did not receive it. Although not statistically significant, there was a trend towards a lower risk of hospitalization in antibody-treated patients. These findings are clinically significant, as lowering mortality and hospitalization due to COVID-19 disease is critical in alleviating the pandemic's burden on the U.S. Additionally, antibodytreated patients also had better odds of fever resolution, dyspnea resolution, and improvement in POX, all indicators of clinical improvement [11].

The binary logistic analysis, which tends to describe the outcome based on baseline patient characteristics without comparing the treatment and the control group, suggested an association of higher mortality with what seems to be markers of more severe disease (for example, time to resolution of shortness of breath) and pro-coagulant activation such as D-dimer.

No serious adverse events occurred in any of the 162 patients in the LY-CoV555 group. Most of the adverse events were mild and transient, including diarrhea, nausea, headache, vomiting, and chills, and one patient with a mild rash. No change of vital signs was detected during the completion of the infusion. Therefore, the side effects related to infusions were relatively minor and were seen in 4.37\% (7/160) of antibody-treated patients.

Originally the US-FDA issued an emergency use authorization (EUA) for monoclonal antibody therapy for the treatment of mild-to-moderate COVID-19 in adult and pediatric patients [29]. However, at the time of this study (November 2020 - January 2021), variants of the COVID-19 virus only had a prevalence of about $5 \%$ in the U.S. In March 2021, prevalence has increased to approximately 20\%. Therefore, as of April 16, 2021, the FDA revoked the single use of LY-CoV555 after Eli Lilly requested to do so in order to prevent possible treatment failures. Lily released a new dual monoclonal antibody treatment, LY-CoV555 with LY-CoV016 (Etesevimab), which confers better protection against U.K. variant strains that are now predominant in the US [30].

\section{Limitation}

The major limitation of our study is its retrospective design. We also only used LY-CoV555 and not REGNCOV2. Lastly, there are some differences in baseline characteristics of the case and control group.

\section{Conclusions}

Our analysis demonstrates that early treatment with monoclonal antibody LY-CoV555 was associated with decreased odds of mortality among high-risk patients in LTCFs with mild-to-moderate COVID-19 infection. Although not statistically significant, there was a trend toward a lower risk of hospitalization in LY-CoV555treated patients. Further studies are needed to confirm these associations.

\section{Additional Information \\ Disclosures}

Human subjects: Consent was obtained or waived by all participants in this study. Animal subjects: All authors have confirmed that this study did not involve animal subjects or tissue. Conflicts of interest: In compliance with the ICMJE uniform disclosure form, all authors declare the following: Payment/services info: All authors have declared that no financial support was received from any organization for the submitted work. Financial relationships: All authors have declared that they have no financial relationships at present or within the previous three years with any organizations that might have an interest in the submitted work. Other relationships: All authors have declared that there are no other relationships or activities that could appear to have influenced the submitted work. 


\section{References}

1. Fauci AS, Lane HC, Redfield RR: Covid-19 - navigating the uncharted . N Engl J Med. 2020, 382:1268-9. 10.1056/NEJMe2002387

2. COVID-19 dashboard by the Center for Systems Science and Engineering (CSSE) at Johns Hopkins University. (2020). Accessed: April 5, 2021:

https://gisanddata.maps.arcgis.com/apps/opsdashboard/index.html\#/bda7594740fd40299423467b48e9ecf6.

3. The New York Times. How many of these 68,000 deaths could have been avoided? . (2020). https://www.nytimes.com/2020/09/05/opinion/sunday/coronavirus-nursing-homes-deaths.html.

4. Borba MGS, Val FFA, Sampaio VS, et al.: Effect of high vs low doses of chloroquine diphosphate as adjunctive therapy for patients hospitalized with severe acute respiratory syndrome coronavirus 2 (SARSCoV-2) infection: a randomized clinical trial. JAMA Netw Open. 2020, 3:e208857.

10.1001/jamanetworkopen.2020.8857

5. Spinner CD, Gottlieb RL, Criner GJ, et al.: Effect of remdesivir vs standard care on clinical status at 11 days in patients with moderate COVID-19. A randomized clinical trial. JAMA. 2020, 324:1048-57. 10.1001/jama.2020.16349

6. Ahmed S, Karim MM, Ross AG, et al.: A five-day course of ivermectin for the treatment of COVID-19 may reduce the duration of illness. Int J Infect Dis. 2021, 103:214-6. 10.1016/j.ijid.2020.11.191

7. Rosas IO, Bräu N, Waters M, et al.: Tocilizumab in hospitalized patients with COVID-19 pneumonia [Preprint]. MedRxiv. 2020, 10.1101/2020.08.27.20183442

8. Shang L, Zhao J, Hu Y, Du R, Cao B: On the use of corticosteroids for 2019-nCoV pneumonia . Lancet. 2020, 395:683-4. 10.1016/S0140-6736(20)30361-5

9. Li L, Zhang W, Hu Y, et al.: Effect of convalescent plasma therapy on time to clinical improvement in patients with severe and life-threatening COVID-19: a randomized clinical trial. JAMA. 2020, 324:460-7. 10.1001/jama.2020.10044

10. Ahmad I, Alam M, Saadi R, et al.: Doxycycline and hydroxychloroquine as treatment for high-risk COVID-19 patients: experience from case series of 54 patients in long-term care facilities [Preprint]. MedRxiv. 2020, 10.1101/2020.05.18.20066902

11. Alam MM, Mahmud S, Rahman MM, Simpson J, Aggarwal S, Ahmed Z: Clinical outcomes of early treatment with doxycycline for 89 high-risk COVID-19 patients in long-term care facilities in New York. Cureus. 2020, 12:e9658. 10.7759/cureus.9658

12. Hoffmann M, Kleine-Weber H, Schroeder S, et al.: SARS-CoV-2 cell entry depends on ACE2 and TMPRSS2 and is blocked by a clinically proven protease inhibitor. Cell. 2020, 181:271-280.e8. 10.1016/j.cell.2020.02.052

13. Hansen J, Baum A, Pascal KE, et al.: Studies in humanized mice and convalescent humans yield a SARS-CoV2 antibody cocktail. Science. 2020, 369:1010-4. 10.1126/science.abd0827

14. Regeneron. (2020). https://investor.regeneron.com/news-releases/news-release-details/regenerons-covid19-outpatient-trial-prospectively-....

15. Chen P, Nirula A, Heller B, et al.: SARS-CoV-2 neutralizing antibody LY-CoV555 in outpatients with Covid19. N Engl J Med. 2021, 384:229-37. 10.1056/NEJMoa2029849

16. Weinreich DM, Sivapalasingam S, Norton T, et al.: REGN-COV2, a neutralizing antibody cocktail, in outpatients with Covid-19. N Engl J Med. 2021, 384:238-51. 10.1056/NEJMoa2035002

17. Vabret N, Britton GJ, Gruber C, et al.: Immunology of COVID-19: current state of the science. Immunity. 2020, 52:910-41. 10.1016/j.immuni.2020.05.002

18. Zhou Y, Fu B, Zheng X, et al.: Pathogenic T cells and inflammatory monocytes incite inflammatory storm in severe COVID-19 patients. Natl Sci Rev. 2020, 7:nwaa041. 10.1093/nsr/nwaa041

19. Conti P, Ronconi G, Caraffa A, Gallenga CE, Ross R, Frydas I, Kritas SK: Induction of pro-inflammatory cytokines (IL-1 and IL-6) and lung inflammation by coronavirus-19 (COV-19 or SARS-CoV-2): antiinflammatory strategies. J Biol Regul Homeost Agents. 2020, 34:327-31. 10.23812/CONTI-E

20. Levi M, Thachil J, Iba T, Levy JH: Coagulation abnormalities and thrombosis in patients with COVID-19 . Lancet Haematol. 2020, $7:$ e438-40. 10.1016/S2352-3026(20)30145-9

21. Tang N, Li D, Wang X, Sun Z: Abnormal coagulation parameters are associated with poor prognosis in patients with novel coronavirus pneumonia. J Thromb Haemost. 2020, 18:844-7. 10.1111/jth.14768

22. Anderson EJ, Rouphael NG, Widge AT, et al.: Safety and immunogenicity of SARS-CoV-2 mRNA-1273 vaccine in older adults. N Engl J Med. 2020, 383:2427-38. 10.1056/NEJMoa2028436

23. Dagan N, Barda N, Kepten E, et al.: BNT162b2 mRNA Covid-19 vaccine in a nationwide mass vaccination setting. N Engl J Med. 2021, 384:1412-23. 10.1056/NEJMoa2101765

24. Rogers R, Shehadeh F, Mylona EK, et al.: Convalescent plasma for patients with severe COVID-19: a matched cohort study. Clin Infect Dis. 2020, ciaa1548. 10.1093/cid/ciaa1548

25. Duan K, Liu B, Li C, et al.: Effectiveness of convalescent plasma therapy in severe COVID-19 patients . Proc Natl Acad Sci U S A. 2020, 117:9490-6. 10.1073/pnas.2004168117

26. Joyner MJ, Carter RE, Senefeld JW, et al.: Convalescent plasma antibody levels and the risk of death from Covid-19. N Engl J Med. 2021, 384:1015-27. 10.1056/NEJMoa2031893

27. Pinto D, Park YJ, Beltramello M, et al.: Cross-neutralization of SARS-CoV-2 by a human monoclonal SARSCoV antibody. Nature. 2020, 583:290-5. 10.1038/s41586-020-2349-y

28. Marovich M, Mascola JR, Cohen MS: Monoclonal antibodies for prevention and treatment of COVID-19 . JAMA. 2020, 324:131-2. 10.1001/jama.2020.10245

29. U.S. Food and Drug Administration. Coronavirus (COVID-19) update: FDA authorizes monoclonal antibody for treatment of COVID-19. (2020). Accessed: November 10, 2020: https://www.fda.gov/news-events/pressannouncements/coronavirus-covid-19-update-fda-authorizes-monoclonal-antibody-tr....

30. U.S. Food and Drug Administration. Coronavirus (COVID-19) update: FDA authorizes monoclonal antibody for treatment of COVID-19. (2021). Accessed: April 17, 2021: https://www.fda.gov/news-events/pressannouncements/coronavirus-covid-19-update-fda-revokes-emergency-use-authorizati.... 